\title{
Frequency-Domain Identification of Continuous-Time Output Error Models from Sampled Data
}

\author{
Jonas Gillberg, Lennart Ljung \\ Division of Automatic Control \\ Department of Electrical Engineering \\ Linköpings universitet, SE-581 83 Linköping, Sweden \\ WWW: http://wwW. control.isy.liu.se \\ E-mail: gillberg@isy.liu.se, ljung@isy.liu.se
}

5th November 2004

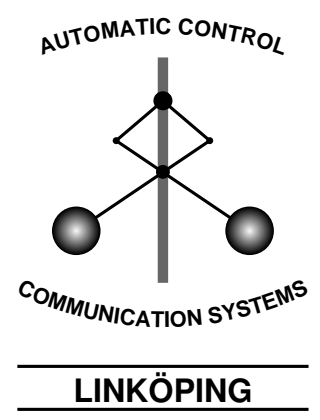

Report no.: LiTH-ISY-R-2643

Submitted to 16th IFAC World Congress, Prague

Technical reports from the Control \& Communication group in Linköping are available at http://www.control.isy.liu.se/publications. 


\begin{abstract}
This paper treats identification of continuous-time output error (OE) models based on sampled data. The exact method for doing this is well known both for data given in the time and frequency domains. This approach becomes somewhat complex, especially for non-uniformly sampled data. We study various ways to approximate the exact method for reasonably fast sampling. While an objective is to gain insights into the non-uniform sampling case, this paper only gives explicit results for uniform sampling.
\end{abstract}

Keywords: continuous-time systems, parameter estimation, continuoustime OE 


\title{
Frequency-Domain Identification of Continuous-Time Output Error Models from Sampled Data
}

\author{
Jonas Gillberg, Lennart Ljung
}

2004-11-05

\begin{abstract}
This paper treats identification of continuous-time output error (OE) models based on sampled data. The exact method for doing this is well known both for data given in the time and frequency domains. This approach becomes somewhat complex, especially for non-uniformly sampled data. We study various ways to approximate the exact method for reasonably fast sampling. While an objective is to gain insights into the non-uniform sampling case, this paper only gives explicit results for uniform sampling.
\end{abstract}

\section{Introduction}

In this contribution we shall discuss identification of possibly grey-box structured linear continuous time models from discrete-time measurements of inputs and outputs. This as such is a well known problem and discussed, e.g. in (Ljung, 1999). Several techniques for identification of continuous time models are also discussed in, among many references, (Rao and Garnier, 2002), (Unbehauen and Rao, 1990), (Mensler, 1999).

The "optimal" solution is well known as a Maximum-likelihood (ML) formulation. It consist of computing the Kalman filter predictions of the output at the sampling instants by sampling the continuous time model over the sampling instants. These predictions are functions of the parameters in the continuous time model and by minimizing the sum of squared prediction errors with respect to the parameters, the Maximum likelihood estimate is obtained in case of Gaussian disturbances. For equidistantly sampled data, this method is also implemented in the System Identification Toolbox, (Ljung, 2003).

No method can be better, in theory, asymptotically as the number of data tends to infinity, than this maximum likelihood method. However, it may encounter numerical problems at fast sampling, and it may be computationally demanding for irregularly sampled data.

We shall therefore here investigate some approximations based on frequency domain data that may be useful alternatives to the basic ML method. For relevant references on frequency domain identification see, e.g. (Pintelon and Schoukens, 2001). While an important objective for us is to gain insights into 
the case of irregular sampling we shall here concentrate on equidistant sampling to bring out the essential issues.

When we describe some expressions and implications for identification we shall occasionally use code from the MATLAB toolbox (Ljung, 2003).

\section{Notation and some basic facts}

\subsection{The model and the transformed signals}

The problem is to estimate the parameters $\theta$ in a continuous time transfer function

$$
y_{u}(t)=G_{c}(p, \theta) u(t)
$$

The output is observed at sampling instances $t_{k}$ with some measurement noise

$$
y\left(t_{k}\right)=y_{u}\left(t_{k}\right)+e(k)
$$

As mentioned in the introduction, we shall throughout this paper consider equidistant sampling with sampling interval $T_{s}: t_{k}=k T_{s}$. The output noise term $e$ is assumed to be Gaussian white noise. For the input $u$ and the output $y$ we define the continuous time Fourier transforms, restricted to an observation interval $[0 \mathrm{~T}]$ :

$$
Y_{c}(i \omega)=\int_{0}^{T} y(t) e^{-i \omega t} d t
$$

and analogously for $U_{c}(i \omega)$. From the sampled data of $y(t), y\left(k T_{s}\right), k=1,2, \ldots, N\left(N T_{s}=\right.$ $T$ ) we can define the discrete-time Fourier transform

$$
Y_{d}\left(e^{i \omega T_{s}}\right)=T_{s} \sum_{k=1}^{N} y\left(k T_{s}\right) e^{-i \omega k T_{s}}
$$

and similarly for $U_{d}\left(e^{i \omega T_{s}}\right)$.

\subsection{Relations between continuous and discrete-time Fourier transforms}

If signals at equidistant sample points $u\left(k T_{s}\right)$ are known, the discrete time Fourier transform (4) can be readily computed. If also the intersample behavior of the signal is known, the continuous time signal can be reconstructed and the continuous time Fourier transform (3) can be determined.

For example if the signal is constant between the sampling points ("zeroorder hold", zoh) it is straightforward to establish that

$$
\begin{aligned}
U_{c}(i \omega) & =H_{T_{s}}(i \omega) U_{d}\left(e^{i \omega T_{s}}\right) \\
H_{T_{s}}(i \omega) & =\frac{1-e^{-i \omega T_{s}}}{T_{s} i \omega}
\end{aligned}
$$

Similarly, if the signal is piecewise linear, connecting the sampled valued ("first order hold") we have

$$
H_{T_{s}}^{f}(i \omega)=e^{-i \omega T_{s}}\left(\frac{e^{i \omega T_{s}}-1}{T_{s} i \omega}\right)^{2}
$$


If the signal is band-limited with all power below the Nyquist frequency, which corresponds to the case where the intersample values are obtained by trigonometric interpolation we have the simple relationship

$$
U_{c}(i \omega)= \begin{cases}U_{d}\left(e^{i \omega T_{s}}\right), & |\omega| \leq \pi / T_{s} \\ 0 & |\omega|>\pi / T_{s}\end{cases}
$$

The general relationship between $Y_{c}$ and $Y_{d}$ comes from Poisson's summation formula:

$$
Y_{d}\left(e^{i \omega T_{s}}\right)=\sum_{k=-\infty}^{\infty} Y_{c}\left(i \omega+i \frac{2 \pi}{T_{s}} k\right)
$$

\subsection{Sampling the model}

If the intersample behavior of the input is known, it is easy to compute a discrete-time pulse transfer function

$$
G_{d}(q, \theta)
$$

that describes how equally sampled input-output data (sampling period $T_{s}$ ) relate. Formulas for $G_{d}$ based on a state-space representation of the transfer function $G_{c}(p, \theta)$ are given in all relevant textbooks, like (Åström and Wittenmark, 1984).

Direct expressions for $G_{d}$ can also be given. If the input is zero-order hold (constant between samples) we have

$$
G_{d}\left(e^{i \omega T_{s}}\right)=\left(\frac{1-e^{-i \omega T_{s}}}{T_{s}}\right) \sum_{k=-\infty}^{\infty} \frac{G_{c}\left(i \omega+i \frac{2 \pi}{T_{s}} k\right)}{i \omega+i \frac{2 \pi}{T_{s}} k} .
$$

We refer to the discussion around Theorems 4.1 and 4.2 in (Åström and Wittenmark, 1984), and to section 8.3.1 in (Gillberg, 2004) for more details on this. (See also Problem 2G.4 in (Ljung, 1999).) The similarity with the Poisson summation formula for Fourier transforms in (8) is also striking.

\subsection{Maximum likelihood estimates}

Suppose that we have available values $Y_{d}\left(e^{i \omega_{k} T_{s}}\right)$ and $U_{d}\left(e^{i \omega_{k} T_{s}}\right), k=1,2, \ldots, N_{\omega}$ of the discrete-time Fourier transforms (4). Suppose the values of $Y$ are independent at different frequencies and that we neglect transient (non-periodic) effects. Then the ML-procedure for estimating the parameters is

$$
V_{d}(\theta) \triangleq \sum_{k=1}^{N_{\omega}}\left|Y_{d}\left(e^{i \omega_{k} T_{s}}\right)-G_{d}\left(e^{i \omega_{k} T_{s}}, \theta\right) U_{d}\left(e^{i \omega_{k} T_{s}}\right)\right|^{2}
$$

If, on the other hand values $Y_{c}\left(i \omega_{k}\right)$ and $U_{c}\left(i \omega_{k}\right), k=1,2, \ldots, N_{\omega}$ of the continuous-time Fourier transforms (3), the ML method under the same assumptions is

$$
V_{c}(\theta) \triangleq \sum_{k=1}^{N_{\omega}}\left|Y_{c}\left(i \omega_{k}\right)-G_{c}\left(i \omega_{k}, \theta\right) U_{c}\left(i \omega_{k}\right)\right|^{2}
$$


See, e.g. page 230 in (Ljung, 1999). Independence of the Fourier transforms at different frequencies is discussed in detail in e.g. (Brillinger, 1981), Chapter 5 and in (Gillberg, 2004), Chapter 3. The bottom line is that the frequencies should be separated by an interval that is $2 \pi / T$.

\section{The crux: Getting $Y_{c}$ from $Y_{d}$}

The most direct way of estimating the continuous time model $G_{c}(i \omega, \theta)$ is to use the criterion (12). This requires the continuous time Fourier transforms. If the intersample behavior of the input is known, which is not unreasonable, $U_{c}$ can be computed from the sampled input values, e.g. by any of the methods described in Section 2.2. It could be a bigger challenge to find $Y_{c}$. If the intersample behavior of the input is known, and if the system is known, the intersample behavior of the output can also be calculated, and hence $Y_{c}$ be determined. But note that this can be done only if the system is known! We shall in Sections 5 and 6 discuss approximations of this idea, that do not require knowledge of the true system.

Note that $y$ has contributions both from $u$ and from the noise $e$. For the estimation result it is only necessary to obtain a correct treatment of contribution $y_{u}$. For example if the input $u$ is band-limited, so will $y_{u}$ be, and the simple formulas (7) can be applied both to input and output.

The simplest approach to this problem is to push one's luck and assume that the data are sampled so fast that they can be considered as band-limited, i.e. using (7). If the true system is of low pass character, this assumption may be more plausible for the output than for the input. In MATLAB terms this would mean

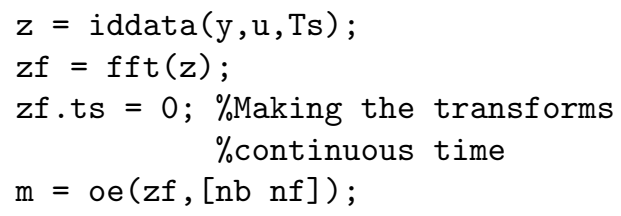

We will label this Approach 1.

\section{Using the summation formula for $G_{d}$}

The "correct" method is, as mentioned in the introduction to use (11) with a carefully sampled transfer function $G_{d}$. For this, one could use the traditional state-space based formulas or the infinite sum (10). This sum could be split into the central term $(k=0)$ and the remaining infinite number of terms, which can be written

$$
G_{d}\left(e^{i \omega T_{s}}, \theta\right)=G_{c}(i \omega, \theta) H_{T_{s}}(i \omega)+R(i \omega)
$$

where $H_{T_{s}}$ is given by (5b). 


\subsection{Using just the central term}

If the system is of low pass character and $T_{s}$ is small $R(i \omega)$ will be small. For example, consider the system

$$
G_{c}(p)=\frac{1}{s^{2}+3 s+2}
$$

and $T_{s}=1$, which is not very fast sampling, then the terms for $k= \pm 1$ in (10) at $\omega=0$ is about $3 \%$ of the central term and the terms for $k= \pm 2$ about $10^{-3}$ of the central term.

It could thus be a reasonable approximation to use just the central term in (10) in (11). This gives the criterion

$$
\begin{aligned}
V_{d}(\theta)= & \sum_{k=1}^{N_{\omega}} \mid Y_{d}\left(e^{i \omega_{k} T_{s}}\right) \\
& \quad-\left.G_{c}\left(i \omega_{k}\right) H_{T_{s}}\left(i \omega_{k}\right) U_{d}\left(e^{i \omega_{k} T_{s}}\right)\right|^{2} \\
= & \sum_{k=1}^{N_{\omega}}\left|Y_{d}\left(e^{i \omega_{k} T_{s}}\right)-G_{c}\left(i \omega_{k}\right) U_{c}\left(i \omega_{k}\right)\right|^{2}
\end{aligned}
$$

where the last step follows from (5) for a zoh input. Note the interpretation of this step! We have arrived at a continuous time criterion (12) where the zoh input is correctly translated to continuous time and for the output a bandlimited assumption (7) is used. This is in line with the assumption that the system is low pass in relation to the sampling interval. In MATLAB terms we have

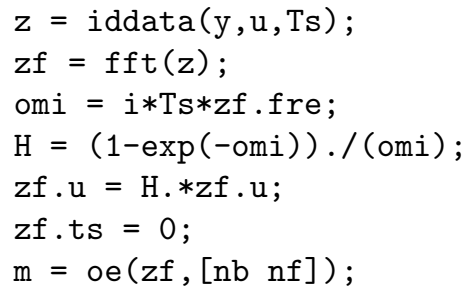

We label this Approach 2.

\subsection{Using more terms}

An obvious variant of this approach is to involve more terms from (10) in (11). If $k= \pm 1, \ldots \pm F$ are included we call this Approach $3-F$. Clearly, as $F \rightarrow \infty$ we approach the "correct" method.

\section{Approximation of $Y_{c}$ at high frequencies}

We mentioned in Section 3 that the correct translation from $Y_{d}$ to $Y_{c}$ must involve the true system. Let us investigate this.

Let $G_{d}\left(e^{i \omega T_{s}}, \theta\right)$ be the sampled model, using as assumption that the input is zoh. Then we have

$$
Y_{d}\left(e^{i \omega T_{s}}\right)=G_{d}\left(e^{i \omega T_{s}}, \theta\right) U_{d}\left(e^{i \omega T_{s}}\right)
$$


We also have the continuous time relationship

$$
\begin{aligned}
Y_{c}(i \omega) & =G_{c}(i \omega, \theta) U_{c}(i \omega) \\
& =G_{c}(i \omega) H_{T_{s}}(i \omega) U_{d}\left(e^{i \omega T_{s}}\right)
\end{aligned}
$$

using (5). This gives the following relationship for $Y_{c}$ :

$$
\begin{aligned}
Y_{c}(i \omega) & =F\left(i \omega, \theta_{0}\right) Y_{d}\left(e^{i \omega T_{s}}\right) \\
F(i \omega, \theta) & =\frac{G_{c}(i \omega, \theta) H_{T_{s}}(i \omega)}{G_{d}\left(e^{i \omega T_{s}}, \theta\right)}
\end{aligned}
$$

The problem would thus be solved if the function $F\left(s, \theta_{0}\right)$ were known. But it depends on the unknown true parameter $\theta_{0}$. So what can we do? A possibility is to focus on high frequencies. When the break-points in the Bode plot of $G_{c}(i \omega)$ are passed, the system has a roll-off that behaves like a series of integrators

$$
G_{c}(s) \sim \frac{b_{0}}{s^{\ell}}
$$

where $\ell$ is the pole excess of the system, i.e. the denominator degree minus the numerator degree. The idea is thus to compute $F$ in $(18 \mathrm{~b})$ for the model (19). This is a problem that has been studied in connection with sampling zeros, e.g. in (Åström et al., 1984), (Wahlberg, 1988), (Weller et al., 2001). See also (Gillberg and Ljung, 2005) and (Goodwin et al., 2005) at this congress.

For the system (19) the function $F$ takes the form:

$$
F_{c}^{(\ell)}(i \omega)=\frac{\ell !\left(e^{i \omega T_{s}}-1\right)^{\ell+1}}{\left(i \omega T_{s}\right)^{\ell+1}\left(e^{i \omega T_{s}} B_{\ell}\left(e^{i \omega T_{s}}\right)\right)}
$$

where $B_{l}(z)$ are the Euler-Frobenius polynomials (see the references above):

$$
\begin{aligned}
& B_{1}(z)=1 \\
& B_{2}(z)=z+1 \\
& B_{3}(z)=z^{2}+4 z+1 \\
& B_{4}(z)=z^{3}+11 z^{2}+11 z+1
\end{aligned}
$$

We have the following approximation result:

Theorem 5.1 Let $G_{c}(s)$ be a transfer function with pole excess $\ell \geq 1$, and let $G_{d}(z)$ be the sampled counterpart using a zero-order hold input. Let $H_{T}(s)$ be given by (5b), $F(s, \theta)$ by (18b) and let $F_{c}^{(\ell)}(i \omega)$ be defined by (20)-(21). Then

$$
\left|F(i \omega, \theta)-F_{c}^{(\ell)}(i \omega)\right| \leq C T_{s}^{\ell+1}
$$

Proof: Since $\omega$ is below the Nyquist frequency

$$
\frac{G_{c}\left(i \omega+i \frac{2 \pi}{T_{s}} k\right)}{\frac{b_{0}}{\left(i \omega+i \frac{2 \pi}{T_{s}} k\right)^{\ell}}} \rightarrow 1
$$


as $T_{s} \rightarrow 0$ if $k \neq 0$ and $b_{0}$ is defined as in (19). This has the consequence that

$$
\frac{G_{c}(i \omega) H(i \omega)}{G_{d}\left(e^{i \omega T_{s}}\right)} \rightarrow \frac{\frac{G_{c}(i \omega)}{i \omega}}{\frac{G_{c}(i \omega)}{i \omega}+\sum_{k \neq 0} \frac{b_{0}}{\left(i \omega+i \frac{2 \pi}{T_{s}} k\right)^{\ell+1}}} .
$$

as $T_{s} \rightarrow 0$ if we insert (23) in (10). From Lemma 3.2 in (Wahlberg, 1988)

$$
F_{c}^{(\ell)}(i \omega)=\frac{\frac{1}{(i \omega)^{\ell+1}}}{\frac{1}{(i \omega)^{\ell+1}}+\sum_{k \neq 0} \frac{1}{\left(i \omega+i \frac{2 \pi}{T_{s}} k\right)^{\ell+1}}} .
$$

By putting the two previous expressions on a common denominator, we get the the following relation

$$
\frac{G_{c}(i \omega) H(i \omega)}{G_{d}\left(e^{i \omega T_{s}}\right)}-F_{c}^{(\ell)}(i \omega) \rightarrow F_{c}^{(\ell)}(i \omega) R(i \omega) S(i \omega)
$$

where

$$
R(i \omega)=\frac{1-G_{c}(i \omega) \frac{(i \omega)^{\ell}}{b_{0}}}{\frac{G_{c}(i \omega)}{i \omega}+\sum_{k \neq 0} \frac{b_{0}}{\left(i \omega+i \frac{2 \pi}{T_{s}} k\right)^{\ell+1}}}
$$

and

$$
S(i \omega)=\sum_{k \neq 0} \frac{b_{0}}{\left(i \omega+i \frac{2 \pi}{T_{s}} k\right)^{\ell+1}} .
$$

Since $F$ and $R$ are bounded in $\omega, \omega$ is below the Nyquist frequency and the terms of $S$ are bounded as

$$
\left|\frac{1}{\left(i \omega+i \frac{2 \pi}{T_{s}} k\right)^{\ell+1}}\right| \leq\left|\frac{T_{s}^{\ell+1}}{\left(i \omega T_{s}+i 2 \pi k\right)^{\ell+1}}\right| \leq C\left(\frac{T_{s}}{k}\right)^{\ell+1}
$$

if $k \neq 0$, the result

$$
\left|\frac{G_{c}(i \omega) H(i \omega)}{G_{d}\left(e^{i \omega T_{s}}\right)}-F_{c}^{(\ell)}(i \omega)\right| \leq\left|F_{c}^{(\ell)}\right||R||S| \leq C T_{s}^{\ell+1}
$$

follows.

In MATLAB code, using this approach in (12) would give

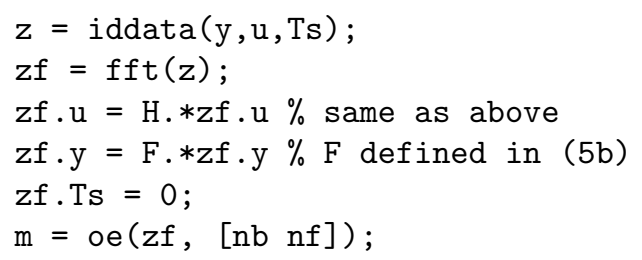

We call this Approach 4. 


\section{Using Poisson's formula}

Yet another approach to (approximately) determine $Y_{c}$ from $Y_{d}$ is to start from the Poisson summation expression (하). This gives

$$
Y_{c}(i \omega)=Y_{d}\left(e^{i \omega T_{s}}\right)-\sum_{k \neq 0} Y_{c}\left(i \omega+i \frac{2 \pi}{T_{s}} k\right)
$$

Note that, for zoh input

$$
\begin{array}{r}
Y_{c}\left(i \omega+i \frac{2 \pi}{T_{s}} k\right)=G_{c}\left(i \omega+i \frac{2 \pi}{T_{s}} k\right) U_{c}\left(i \omega+i \frac{2 \pi}{T_{s}} k\right) \\
=G_{c}\left(i \omega+i \frac{2 \pi}{T_{s}} k\right) H_{T_{s}}\left(i \omega+i \frac{2 \pi}{T_{s}} k\right) U_{d}\left(e^{i \omega T_{s}}\right) \\
=\frac{G_{c}\left(i \omega+i \frac{2 \pi}{T_{s}} k\right)}{i \omega+i \frac{2 \pi}{T_{s}} k}\left(\frac{1-e^{-i \omega T_{s}}}{T_{s}}\right) U_{d}\left(e^{i \omega T_{s}}\right)
\end{array}
$$

using that $e^{i 2 \pi k}=1$. This means that the correction sum term above takes the form

$$
Y_{c}(i \omega)=Y_{d}\left(e^{i \omega T_{s}}\right)-R(i \omega) U_{d}\left(e^{i \omega T_{s}}\right)
$$

where $R$ is defined in (13). Note that $R$ consists of high frequency terms of $G_{c}$ of the kind that were ignored in Approach 2. The idea now is not to ignore these terms (which would be the band-limited output approach 2) but to approximate them with the assumption (19). This has some resemblance with approach 4, but should be more accurate, since the central $(k=0)$ term is kept as the original system and is not approximated with (19). The disadvantage is that the high frequency gain $b_{0}$ in (19) will not be canceled, but will have to be kept as an additional parameter to be estimated.

Now, it is easy to realize that $R(i \omega)$ for the system (19) will be the difference between the sampled frequency function for $b_{0} / s^{l}$ and the central term $b_{0} H_{T_{s}}(i \omega) /(i \omega)^{l}$. Using the sampling result form (Aström et al., 1984), we then obtain

$$
\begin{aligned}
R(i \omega) & \approx b_{0} F_{d c}^{(\ell)}(i \omega) \\
& =b_{0} T_{s}^{l} \frac{B_{l}\left(e^{i \omega T s}\right)}{l !\left(e^{i \omega T s}-1\right)^{l}}-\frac{b_{0}}{(i \omega)^{l}} H_{T_{s}}(i \omega)
\end{aligned}
$$

where $B_{l}$ is given by (21) and $H_{T}$ by $(\overline{5 b}$ )

The resulting approximation will be

$$
Y_{c}(i \omega)=Y_{d}\left(e^{i \omega T_{s}}\right)-b_{0} F_{d c}^{(\ell)}(i \omega) U_{d}\left(e^{i \omega T_{s}}\right)
$$

and the continuous time criterion (12) takes the form

$$
\begin{gathered}
V_{c}(\theta)=\sum_{k=1}^{N_{\omega}} \mid\left(Y_{d}\left(e^{i \omega_{k} T_{s}}\right)-b_{0} F_{d c}^{(\ell)}\left(i \omega_{k}\right) U_{d}\left(e^{i \omega_{k} T_{s}}\right)\right) \\
-\left.G_{c}\left(i \omega_{k}, \theta\right) U_{c}\left(i \omega_{k}\right)\right|^{2}
\end{gathered}
$$




$$
\begin{aligned}
& V_{c}(\theta)=\sum_{k=1}^{N_{\omega}} \mid Y_{d}\left(e^{i \omega_{k} T_{s}}\right) \\
& -\left.\left(b_{0} F_{d c}^{(\ell)}\left(i \omega_{k}\right)+G_{c}\left(i \omega_{k}, \theta\right) H_{T_{s}}\left(i \omega_{k}\right)\right) U_{d}\left(e^{i \omega_{k} T_{s}}\right)\right|^{2}
\end{aligned}
$$

Notice that an alternative interpretation of $(25)$ is that is is based on the sampled expression (11) using an expression for $G_{d}$ being (10) where $G_{c}$ for the noncentral terms $(k \neq 0)$ is replaced by (19). This can also be seen as an illustration of the kinship between the Poisson summation formula (8) and the sampling expression (10)

We call this Approach 5.

\section{$7 \quad$ Numerical illustration}

We shall in this section illustrate how the different approaches perform for some systems with different sampling intervals. In all cases we simulate the system with a binary input with a frequency contents that is adapted to the sampling interval $T_{s}$ :

$\mathrm{u}=\operatorname{iddata}([], \operatorname{idinput}(10000$, 'rbn', . .

$[0 \min (1, \mathrm{Ts})]), \mathrm{Ts})$;

No noise was added to the simulations, and a long data record was chosen, since we wanted to study the bias effects of the approximations involved. The results are given in the following tables.

It should be noted that in practice it may be essential to limit the fit in (11) and $(12)$ to frequencies that do not extend all the way to the Nyquist frequency, since the observations may be less reliable at higher frequencies. Another reason is that $F$ in (20) will tend to infinity at the Nyquist frequency for $l$ being even. (The sampled multi-integrator has a zero at the Nyquist frequency.)

\section{Conclusions}

We have investigated some ways to estimate continuous time models from discrete time data using frequency domain methods. It should be repeated that the "best" way to do this is known: to sample the model, but retaining the continuous parameterization. Some approximate sampling methods have been discussed for the frequency domain approach, that can be seen as ways to approximately calculate the continuous time Fourier transform of the output from its DFT. The conclusion is that the bias caused by these approximation can be quite small, even at sampling rates that are not fast compared to the system dynamics. It is of special interest to see how these approaches may carry over to the case on non-equidistant sampling.

\section{References}

Brillinger, D.R. (1981). Time Series. Holden-Day. San Francisco, CA. 


\begin{tabular}{|l|l|l|l|l|l|}
\hline True/Ts & 0.02 & 0.1 & 0.5 & 1 & 1.5 \\
\hline Appr 1 & & & & & \\
\hline 1 & 0.9753 & 0.8794 & 0.2614 & -0.5413 & -0.3764 \\
0.5 & 0.5137 & 0.5529 & 1.4552 & 1.4623 & 0.5553 \\
2 & 1.9443 & 1.7432 & 1.3928 & 1.5599 & 1.0350 \\
3 & 2.9972 & 2.9670 & 4.8357 & 5.6280 & 3.1000 \\
\hline Appr 2 & & & & & \\
\hline 1 & 1.0002 & 1.0020 & 0.9879 & 0.5292 & -0.1094 \\
0.5 & 0.5002 & 0.5046 & 0.6266 & 1.4389 & 1.0510 \\
2 & 2.0006 & 2.0003 & 1.9033 & 1.3682 & 0.7592 \\
3 & 3.0005 & 3.0046 & 3.1556 & 4.8057 & 4.2858 \\
\hline Appr 4 & & & & & \\
\hline 1 & 0.9999 & 0.9949 & 0.8791 & 0.5541 & 0.1864 \\
0.5 & 0.5000 & 0.5013 & 0.5272 & 0.6046 & 0.5351 \\
2 & 2.0003 & 1.9929 & 1.8353 & 1.4233 & 1.0898 \\
3 & 3.0000 & 2.9986 & 2.9659 & 3.0044 & 2.8335 \\
\hline Appr 5 & & & & & \\
\hline 1 & 1.0047 & 0.9994 & 0.9866 & 0.8223 & 0.4488 \\
0.5 & 0.4956 & 0.5040 & 0.5020 & 0.5244 & 0.4341 \\
2 & 2.0288 & 2.0015 & 1.9760 & 1.6736 & 1.1949 \\
3 & 2.9966 & 3.0093 & 3.0037 & 2.9537 & 2.3787 \\
\hline
\end{tabular}

Table 1: Results for the system $\frac{s+0.5}{s^{2}+2 s+3}$. This system has a pole excess of 1 and a bandwidth of $8.60 \mathrm{rad} / \mathrm{s}$

\begin{tabular}{|l|l|l|l|l|l|}
\hline True/Ts & 0.02 & 0.1 & 0.5 & 1 & 1.5 \\
\hline $\mathrm{F}=1$ & & & & & \\
\hline 1 & 1.0047 & 1.0003 & 1.0093 & 0.9263 & 0.3645 \\
0.5 & 0.4957 & 0.5057 & 0.5416 & 0.6516 & 0.5723 \\
2 & 2.0288 & 2.0019 & 1.9882 & 1.7926 & 1.0915 \\
3 & 2.9968 & 3.0109 & 3.0391 & 3.1560 & 2.7964 \\
\hline $\mathrm{F}=2$ & & & & & \\
\hline 1 & 1.0047 & 1.0000 & 1.0072 & 0.9791 & 0.5087 \\
0.5 & 0.4957 & 0.5050 & 0.5245 & 0.5822 & 0.5119 \\
2 & 2.0288 & 2.0018 & 1.9962 & 1.9114 & 1.3011 \\
3 & 2.9968 & 3.0103 & 3.0215 & 3.0696 & 2.6539 \\
\hline $\mathrm{F}=5$ & & & & & \\
\hline 1 & 1.0047 & 0.9997 & 1.0038 & 1.0036 & 0.6869 \\
0.5 & 0.4957 & 0.5045 & 0.5109 & 0.5341 & 0.4856 \\
2 & 2.0288 & 2.0017 & 1.9998 & 1.9831 & 1.5824 \\
3 & 2.9966 & 3.0098 & 3.0087 & 3.0260 & 2.6876 \\
\hline $\mathrm{F}=10$ & & & & & \\
\hline 1 & 1.0047 & 0.9995 & 1.0021 & 1.0049 & 0.8129 \\
0.5 & 0.4957 & 0.5042 & 0.5056 & 0.5170 & 0.4878 \\
2 & 2.0288 & 2.0016 & 2.0004 & 1.9969 & 1.7655 \\
3 & 2.9967 & 3.0096 & 3.0042 & 3.0127 & 2.7935 \\
\hline
\end{tabular}

Table 2: Results for approach 3 (Section 4.2) for the same system as in Table 1 for different values of $\mathrm{F}$. 


\begin{tabular}{|l|l|l|l|l|l|}
\hline True/Ts & 0.02 & 0.1 & 0.5 & 1 & 1.5 \\
\hline Appr 1 & & & & & \\
\hline 1 & 0.9805 & 0.9096 & 0.6692 & 0.4873 & 0.3584 \\
2 & 1.9550 & 1.7887 & 1.2413 & 0.8993 & 0.7554 \\
3 & 2.9805 & 2.9084 & 2.6481 & 2.4271 & 2.2400 \\
4 & 3.8937 & 3.5078 & 2.2317 & 1.4376 & 1.0490 \\
\hline Appr 2 & & & & & \\
\hline 1 & 1.0017 & 1.0000 & 0.9996 & 0.9857 & 0.9050 \\
2 & 2.0031 & 2.0000 & 1.9993 & 1.9729 & 1.8921 \\
3 & 3.0018 & 3.0000 & 2.9997 & 2.9900 & 2.9346 \\
4 & 4.0061 & 4.0000 & 3.9987 & 3.9458 & 3.7830 \\
\hline Appr 4 & & & & & \\
\hline 1 & 1.0018 & 1.0000 & 1.0015 & 1.0268 & 0.9641 \\
2 & 2.0032 & 1.9998 & 2.0002 & 1.9821 & 1.5172 \\
3 & 3.0019 & 3.0000 & 3.0002 & 3.0044 & 2.8956 \\
4 & 4.0063 & 3.9996 & 4.0013 & 3.9758 & 3.1243 \\
\hline Appr 5 & & & & & \\
\hline 1 & 1.0346 & 0.9993 & 1.0028 & 0.9949 & 0.9900 \\
2 & 2.1001 & 2.0012 & 2.0092 & 1.9930 & 2.0436 \\
3 & 3.0554 & 2.9998 & 3.0040 & 2.9966 & 2.9994 \\
4 & 4.1943 & 4.0038 & 4.0172 & 3.9863 & 4.0891 \\
\hline
\end{tabular}

Table 3: Results for the system $\frac{1}{s^{3}+2 s^{2}+3 s+4}$. This system has a pole excess of 3 and a bandwidth of $2.1 \mathrm{rad} / \mathrm{s}$

\begin{tabular}{|l|l|l|l|l|l|}
\hline Ts & 0.02 & 0.1 & 0.5 & 1 & 1.5 \\
\hline Appr 1 & 4.0081 & 4.0388 & 4.2330 & 4.4837 & 4.7438 \\
\hline Appr 2 & 3.9988 & 3.9935 & 3.9988 & 3.9995 & 4.0020 \\
\hline Appr 4 & 3.9987 & 3.9935 & 3.9988 & 3.9999 & 4.0038 \\
\hline Appr 5 & 3.9444 & 3.9855 & 3.9989 & 3.9951 & 3.9689 \\
\hline
\end{tabular}

Table 4: Estimated values of $a$ for the model $\frac{a}{s^{3}+a^{2} s^{2}+a s+a}$ with true value $a=4$. This system has a pole excess of 3 and a bandwidth of $0.75 \mathrm{rad} / \mathrm{s}$. 
Gillberg, J. (2004). Methods for frequency domain estimation of continuous-time models. Lic. Dissertation Linköping Studies in Science and Technology Thesis No. 1133. Department of Electrical Engineering, Linköping University. SE-581 83 Linköping, Sweden.

Gillberg, J. and L. Ljung (2005). Frequency-domain identification of continuoustime ARMA models from sampled data. In: Proc. of the 16th IFAC World Congress, Prague.

Goodwin, G. C., J. I. Yuz and H. Garnier (2005). Robustness issues in continuous-time system identification from sampled data. In: Proc. of the 16th IFAC World Congress, Prague.

Ljung, L. (1999). System Identification - Theory for the User. 2nd ed.. PrenticeHall. Upper Saddle River, NJ.

Ljung, L. (2003). System Identification Toolbox for use with MATLAB. Version 6. 6th ed.. The MathWorks Inc. Natick, MA.

Mensler, M. (1999). Analyse et etude comparative de methodes d'identification des systemes a representation continue. PhD thesis. Centre de Recherche en Automatique de Nancy, Universite Henri Poincare, Nancy.

Pintelon, R. and J. Schoukens (2001). System Identification - A Frequency Domain Approach. IEEE Press. Piscataway, NJ.

Rao, G. P. and H. Garnier (2002). Numerical illustrations of the relevance of direct continuous-time model identification. In: Proc. of the 15th IFAC World Congress, Barcelona.

Åström, K. J. and B. Wittenmark (1984). Computer Controlled Systems. Prentice-Hall. Englewood Cliffs.

Åström, K.J., P. Hagander and J. Sternby (1984). Zeros of sampled systems. Automatica 20(1), 31-38.

Unbehauen, H. and G. P. Rao (1990). Continuous-time approaches to system identification - a survey. Automatica 26(1), 23-35.

Wahlberg, B. (1988). Limit results for sampled systems. International Journal of Control 48(3), 1267-1283.

Weller, S. R., W. Moran, B. Ninnes and A. D. Pollinton (2001). Sampling zeros and the Euler-Frobenius polynomials. IEEE Transactions on Automatic Control 46(2), 340-343. 


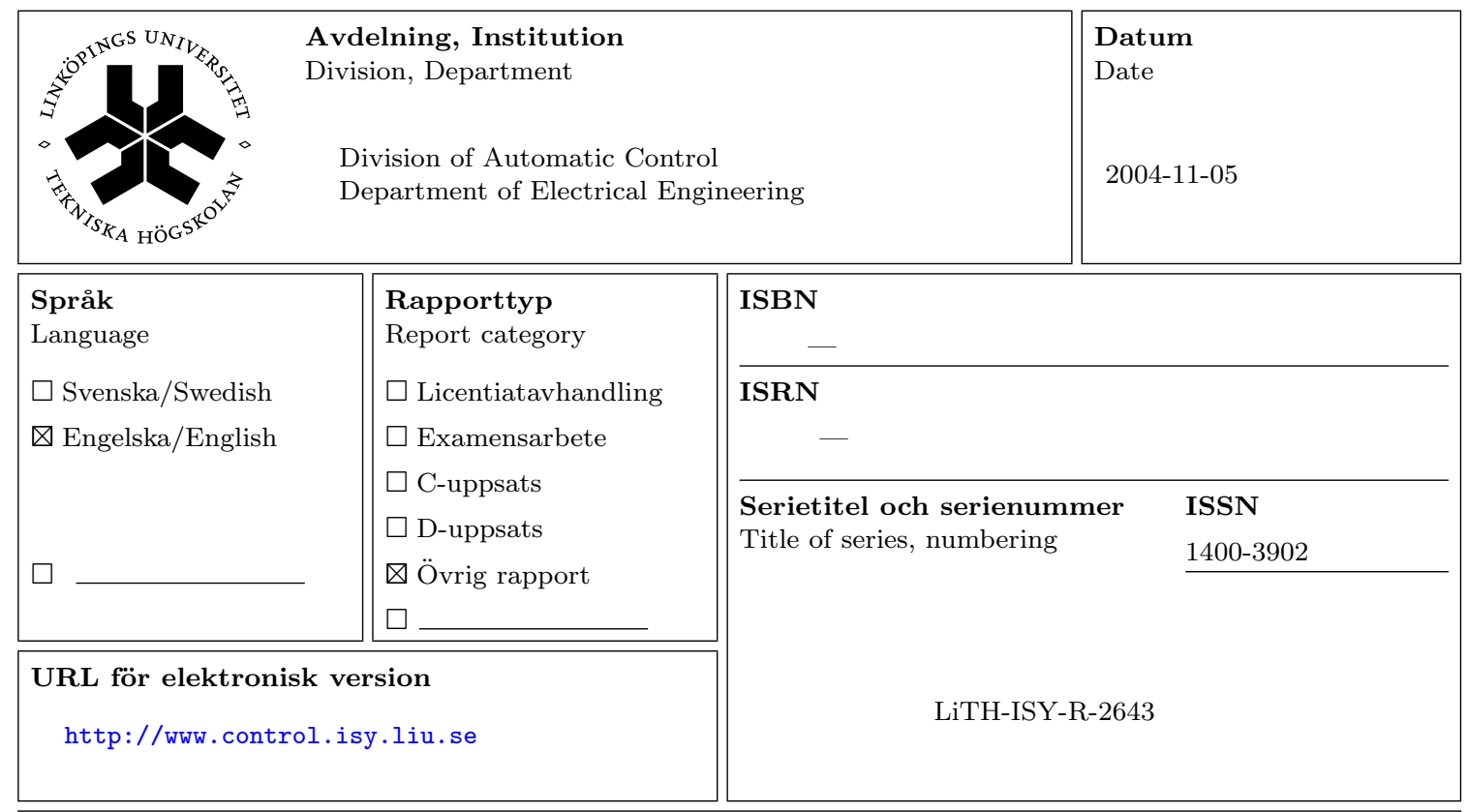

Titel Frequency-Domain Identification of Continuous-Time Output Error Models from Sampled Data

Författare Jonas Gillberg, Lennart Ljung

Author

Sammanfattning

Abstract

This paper treats identification of continuous-time output error (OE) models based on sampled data. The exact method for doing this is well known both for data given in the time and frequency domains. This approach becomes somewhat complex, especially for non-uniformly sampled data. We study various ways to approximate the exact method for reasonably fast sampling. While an objective is to gain insights into the non-uniform sampling case, this paper only gives explicit results for uniform sampling.

Nyckelord

Keywords continuous-time systems, parameter estimation, continuous-time OE 